\title{
Template-Guided Versus Undirected Written Medical Documentation: A Prospective, Randomized Trial in a Family Medicine Residency Clinic
}

Sharon Mulvehill, MD, Gregory Schneider, MD, Cassie Murphy Cullen, PhD, Shelley Roaten, MD, Barbara Foster, PhD, and Anne Porter, BS

Purpose: To compare a template-driven medical documentation system to undirected handwritten documentation and determine whether the template (1) decreases physician evaluation time, (2) increases gross billing, and (3) increases physician satisfaction with the documentation process.

Methods: A prospective randomized trial of documentation with a template system (T-System for Primary Care, Dallas, TX) versus undirected handwritten documentation was conducted in 2 separate teams of a single family medicine residency program. After training, one team used the template system and the other team used undirected written documentation. Clinic visit duration was recorded. Medical records were evaluated by a blinded professional coder to assign an evaluation/management code. Clinic visit duration and coding level differences were evaluated using an independent $t$ test. At the conclusion of the study, residents completed a questionnaire to determine physician satisfaction with the documentation tool. Survey responses were on a -2 to +2 Likert scale. Means and standard deviations are reported.

Results: A total of 1339 patients were included in the analysis of patient visits. There was no significant difference in clinic time between the template system and the written documentation visits. The mean visit time was 1.75 hours for both teams. For the analysis of gross billing, 1237 charts were included. The mean billing amount for written documentation was $\$ 150$ and for the template system it was $\$ 163$ - a statistically significant difference. The physicians' surveys favored continuing to use the template documentation method.

Conclusions: The template medical documentation system compared with undirected written documentation produced a significantly higher bill for the visit, yielding no differences in evaluation time, and was overall positively received by the residents and faculty. (J Am Board Fam Pract 2005;18: 464-9.)

Quality care depends on a clear and complete record, thus making medical documentation an intrinsic component of every patient encounter. There are many reasons to improve the quality of the medical record. Prior studies have suggested that the quality of documentation correlates with

Submitted 25 January 2005; revised 8 June 2005; accepted 13 June 2005.

From the University of Texas Southwestern Family Medicine Residency Program, the University of Texas Southwestern Medical Center at Dallas.

Funding: This study was supported by a grant from TSystem for Primary Care (Dallas, TX).

Conflict of interest: none declared.

Corresponding author: Sharon Mulvehill, MD, Department of Family Medicine, 6303 Harry Hines Boulevard, Dallas, TX 75390-9165 (e-mail: smulve@parknet.pmh.org).

This study was a poster presentation at the 2004 Scientific Assembly of the American Academy of Family Physicians, 13-17 October 2004, in Orlando, FL. the quality of medical care. ${ }^{1,2}$ Quality improvement projects, research, and legal issues all rely on a complete accurate record. Coding, billing, and reimbursement depend on accurate documentation in specific areas. The process of recording a patient visit takes time, thereby affecting physician productivity and income. Time spent by physicians completing records can lengthen their day and create frustration. Family physicians use many different documentation methods today in their practices. These include undirected written charts, templatebased written charts, dictation, and electronic medical records. Recent policies within the American Academy of Family Physicians, other medical specialty organizations, and from the federal government have called for a transition to an electronic medical record (EMR). ${ }^{3,4}$ Recent surveys estimate 
the usage of a full electronic medical record to be $15 \%$. Cost and difficulty choosing an appropriate system are barriers to many physicians. ${ }^{5-7}$

Research is needed to evaluate both EMR and other documentation methods. Results on paper documentation research may offer improvements applicable to many family medicine practices. Lessons learned evaluating paper documentation methods may translate to use on an EMR platform.

The template system chosen for this study is T-System for Primary Care. It is a template-generated documentation system that was first designed for emergency care in 1996. Since then it has been used extensively across the United States. It is currently the documentation method for almost $40 \%$ of all emergency department (ED) visits in the United States and exists in both a paper and an integrated EMR format. The paper format was adapted to primary care in 2001 and is currently used by over a thousand providers. The cost of implementation is currently a $\$ 500$ initial fee and then $\$ 2200$ per year per provider. To study the effects of the template documentation system, we designed a prospective randomized controlled study. This study hypothesizes that the template system will (1) decrease physician evaluation time, (2) increase coding and reimbursement levels, and (3) improve physician satisfaction with the documentation record.

\section{Methods}

The study was performed in an inner city, countyowned, hospital-operated family medicine residency clinic. There are 2 separate teams that draw patients indiscriminately from the same undifferentiated patient population. The 2 teams are in separate but adjacent office space on the same floor of a professional office building. Both teams see equal volume. New patients are assigned to one of the 2 teams based on appointment availability. Thereafter patients are seen by the same team to which they were originally assigned. Residents are assigned to either team at the beginning of their residency and stay in that team through their 3 years of training. A total of 16 residents participated in either the template group or the control group. The clinic treats approximately $10,000 \mathrm{pa}-$ tients per year.

One team was randomly chosen by coin toss to be the study team. The residents assigned to that team received a 1-hour training session on the template system from a company representative. All faculty physicians attended the training. The faculty worked in the same team for the duration of the study with few exceptions. Nurses and medical assistants attended the same training session because they are rotated between teams. The templates were used approximately 6 weeks before the start of data collection.

Three template systems were reviewed before selecting one for use in the study. The selected system was chosen because of higher volume of use and greater customer support. For examples of this template system, please visit http://www.tsystem. com/Template-Systems/primary-care.asp. The template system we used is a series of 40 templates based on chief complaint. Each template includes specific symptoms based on the chief complaint(s). Additional complaints are addressed in a small narrative section. By a series of circles, checks, and backslashes the physician documents the presence or absence of each finding. A tailored review of systems is provided, and there are areas for past medical/social/family history. Relevant physical findings are listed on the template, and they are checked or backslashed to denote the presence or absence. Each of the above sections includes some blank lines for additional information. Shaded areas denote information required for higher levels of service.

There were 2 primary outcomes measured: time of physician evaluation and billing amount. A secondary outcome was physician satisfaction. Physician evaluation time was calculated using the time the patient was placed in the room and the clinic discharge time. A power analysis indicated that a sample size of 650 patients per team had $88 \%$ power to detect a 7 -minute reduction in time (a clinically relevant number). Based on current clinic volume, a study period of 6 weeks was chosen.

To analyze billing amount, an independent trained and certified coder was hired to determine the billing amounts for all patient visits included in the study. The 1995 Health Care Financing Administration (HCFA) guidelines were used. These guidelines are often used in practice because they are less complex than a 1997 revision. The coder was given no details regarding the nature of the study nor was he shown the actual codes assigned by the family medicine department. Based on the 
previously published results, a sample size of 650 has a power of $80 \%$ to detect a $19 \%$ billing increase (an effect size of 0.16).

Both physician evaluation and billing amount were checked for normality and outliers. An independent $t$ test was used to compare both the average examination room time of the 2 teams and the average billing amount across the 2 teams.

At the conclusion of the study a survey was given to the residents of the team using the template to measure physician satisfaction. Permission to use the survey tool was granted by the American College of Emergency Physicians and had been used in a previous study. ${ }^{8}$ Ten questions were administered using a 5 -point Likert scale, with responses ranging from strongly disagree to strongly agree. A set of 3 different questions was asked of the faculty physicians using the same Likert scale. The mean response was calculated. Because this is an unvalidated opinion survey, the comparative analysis was considered secondary, and power was not determined a priori.

All analyses and graphs were performed on SPSS 12.0 (SPSS Inc., Chicago, IL). Institutional Review Board permission was granted before onset of data collection.

\section{Results}

The control team included 626 patient visits with a mean billing amount of $\$ 149.63$ (95\% CI, 145.8 to 153.5), whereas the template team included 611 patient visits with a mean billing amount of $\$ 163.38$ (95\% CI, 159.0 to 167.7$)$. The gross difference is $\$ 13.75$ (95\% CI, 8.0 to 19.5). An independent $t$ test indicates that the gross billing amount is statistically significant across the 2 teams for the independent blinded standard coder $(t=4.67, d f=1235)$ (Figure 1). Table 1 shows the frequency for common billing codes by team. The 2 teams saw a total of 1339 patients over the specified time period. Of these, 1237 patient records were sent to the independent coder. That indicates that 102 medical records were lost to coding. Because of blinding, we were unable to determine which patients were missing the coder information, but the loss was equally distributed across the 2 teams.

Patient visit time, based on time in room to discharge time, showed no significant difference between the control and template team. There were 694 patients in the control team and 645 patients in the template team. The mean visit time was 1.75 hours for both teams (95\% CI about the zero difference is -0.65 to +0.65 ) (Figure 2).

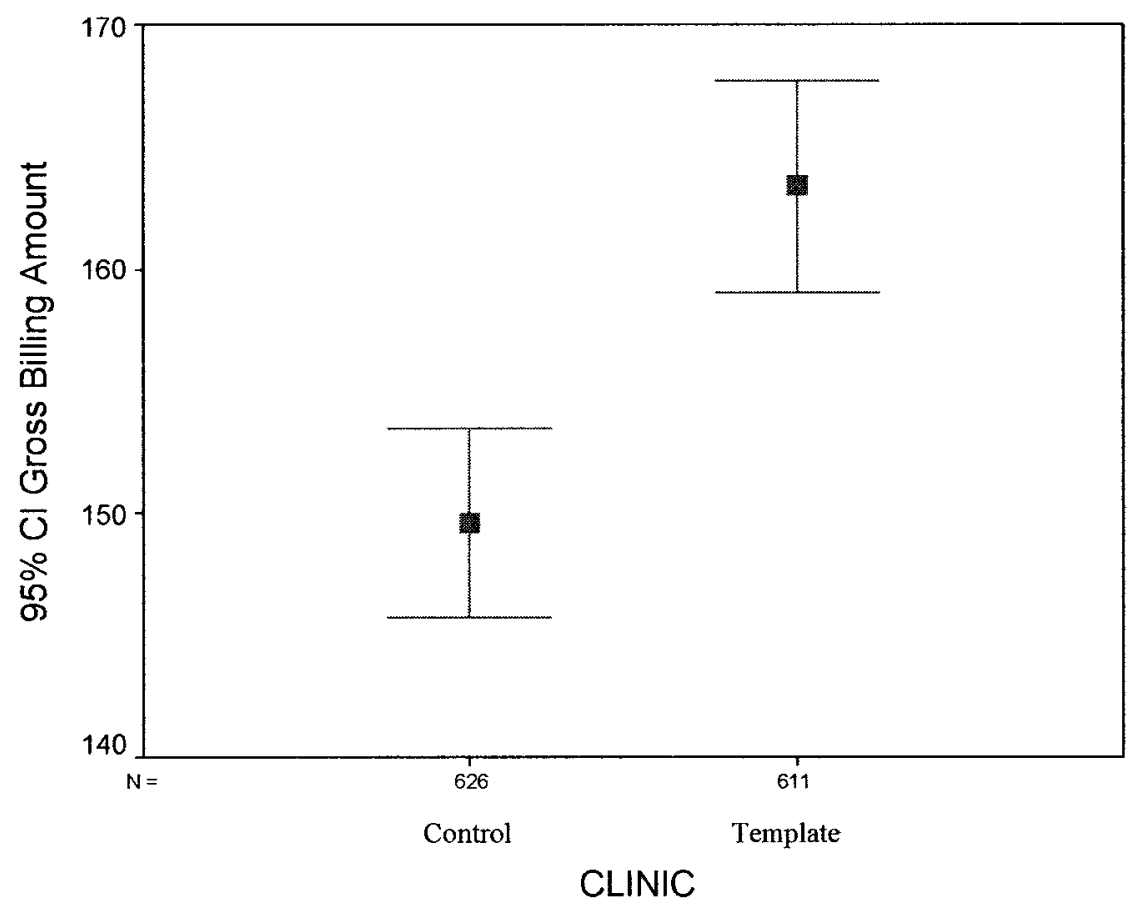

Figure 1. Gross Billing Amount 
Table 1. Number of Patients (with percentage of all codes) by Common Billing Codes and Team

\begin{tabular}{|c|c|c|c|c|c|c|c|c|c|}
\hline & 99203 & 99204 & 99205 & 99212 & 99213 & 99214 & 99215 & $\begin{array}{c}\text { Total of } \\
\text { These Codes }\end{array}$ & $\begin{array}{l}\text { Total of } \\
\text { All Codes }\end{array}$ \\
\hline Template team & $19(3 \%)$ & $18(3 \%)$ & $14(2 \%)$ & $56(9 \%)$ & $104(17 \%)$ & $283(46 \%)$ & $27(4 \%)$ & $521(85 \%)$ & 611 \\
\hline Control team & $20(3 \%)$ & $11(2 \%)$ & $3(<1 \%)$ & $77(12 \%)$ & $168(27 \%)$ & $247(39 \%)$ & $18(3 \%)$ & $544(87 \%)$ & 626 \\
\hline
\end{tabular}

The data for each survey item is displayed in Table 2 for faculty and in Table 3 for residents. Question 1 of the faculty survey was an overall question relating to the continued use of the template system. The average score from the faculty was 1.0 on a scale from -2 to +2 , where -2 indicates a response of strongly disagree and +2 indicates a strongly agree response. Question 10 of the resident survey was also a question on continuing the use of the template, and the average response was 0.5 on the same scale.

\section{Conclusions}

We chose to study a paper-based documentation method for many reasons. Our institution is not ready to implement electronic medical records in the family medicine residency clinic. The main barrier is cost. Although EMR will probably be adopted sometime, there is no specific target date, and we wanted to improve our documentation method now. A template-driven medical documentation system is an available product with a reasonable start up cost, and minimal training time is required. Template systems specifically include issues relevant to the chief complaint. They can remind physicians to inquire about and document important aspects of the visit. This can be an advantage, especially in a training environment. Conversely, a template is a structured format that cannot include all variables in treating a patient.

We chose to measure the time the patient was placed in the room until the patient was discharged to represent the clinic visit time. This time was measurable in a reliable way in our clinic, yet included many variables other than the physician encounter. Our data showed no significant difference in clinic times between the 2 teams. This demonstrates that the template system can be adopted with minimal training time (1 hour) and neither adversely affects nor improves productivity.



Figure 2. Mean Patient Visit Time 
Table 2. Faculty Satisfaction Survey*

\begin{tabular}{lcc}
\hline $\begin{array}{l}\text { Survey Statement } \\
\text { 1. I would like to continue using the T-System } \\
\text { for Primary Care. }\end{array}$ & $\begin{array}{c}\text { Response† } \\
(\mathrm{N}=9)\end{array}$ \\
$\begin{array}{l}\text { 2. I believe the residents are faster with the } \\
\text { T-System for Primary Care. }\end{array}$ & 0.38 & $(0.54)$ \\
$\begin{array}{l}\text { 3. I believe the residents are more accurate } \\
\text { with the T-System for Primary Care. }\end{array}$ & $0.00 \quad(0.93)$
\end{tabular}

* Scale from -2 to +2 where -2 is strongly disagree and +2 is strongly agree.

${ }^{\dagger}$ Mean response with standard deviation in parentheses.

The coding levels shown are representative of our experience in an inner city, county hospital clinic (no lower level new patient codes-99201, 99202). There are few simple visits for new patients; they often present with a list of complaints. In addition there were few high level codes for new patients (99205). Higher level of service is more

Table 3. Resident Satisfaction Survey*

\begin{tabular}{|c|c|c|}
\hline \multirow{2}{*}{$\begin{array}{l}\text { Survey Statement } \\
\text { 1. I see patients more quickly using the } \\
\text { T-System for Primary Care rather than } \\
\text { standard charts. }\end{array}$} & \multicolumn{2}{|c|}{$\begin{array}{l}\text { Response† } \\
(\mathrm{N}=8)\end{array}$} \\
\hline & 0.50 & $(0.93)$ \\
\hline $\begin{array}{l}\text { 2. I collect a more complete database using } \\
\text { the T-System for Primary Care rather } \\
\text { than the standard charts. }\end{array}$ & 0.00 & $(0.93)$ \\
\hline $\begin{array}{l}\text { T-System for Primary Care } \\
\text { to use. } \neq\end{array}$ & -0.38 & $(0.74)$ \\
\hline $\begin{array}{l}\text { 4. Documentation takes less time using the } \\
\text { T-System for Primary Care rather than } \\
\text { the standard charts. }\end{array}$ & 0.50 & $(0.93)$ \\
\hline $\begin{array}{l}\text { 5. I spend more time with the patient when I } \\
\text { use the T-System for Primary Care. }\end{array}$ & 13 & $(0.64)$ \\
\hline $\begin{array}{l}\text { 6. The patients are concerned about the } \\
\text { checklist approach of the T-System for } \\
\text { Primary Care. } \neq\end{array}$ & -0.75 & $(0.46)$ \\
\hline $\begin{array}{l}\text { 7. The T-System for Primary Care charts } \\
\text { guide my history and physical when I see } \\
\text { a patient. }\end{array}$ & 0.25 & $(0.89)$ \\
\hline $\begin{array}{l}\text { 8. The T-System for Primary Care charts } \\
\text { lead me away from the correct workup. } \neq\end{array}$ & -0.50 & $(0.54)$ \\
\hline $\begin{array}{l}\text { 9. The differential list at the bottom of the } \\
\text { T-System for Primary Care charts has } \\
\text { helped me make a diagnosis that I would } \\
\text { not have otherwise considered. }\end{array}$ & -0.25 & $(0.88)$ \\
\hline $\begin{array}{l}\text { 10. I would like to continue using the } \\
\text { T-System for Primary Care. }\end{array}$ & 0.50 & 3) \\
\hline
\end{tabular}

* Scale from -2 to +2 where -2 is strongly disagree and +2 is strongly agree.

$\dagger$ Mean response with standard deviation in parentheses.

‡ Question appears as stated. A negative response indicates a favorable impression of the template system. difficult to deliver and document in new patients because documentation guidelines for new patients are more extensive at the higher level of service.

Regarding the coding data, there was significant improvement in overall billing in the team using the template system. The HCFA guidelines are a complex set of directives regarding required documentation for each evaluation and management code. Prior studies have determined that both under and over coding are common errors using these guidelines. ${ }^{78}$ In addition, expert coders frequently do not agree when assigning a level of service. ${ }^{8}$ This is worrisome because an accusation that a physician is coding improperly can lead to audits and penalties. Complete records with required documentation should yield better billing data by preventing both internal and payer down coding. The mean professional bill was $\$ 162$ for the template team and $\$ 149$ for the undirected charting groups, using the 1995 HCFA Guidelines.

Physician satisfaction survey results show that both residents and faculty physicians involved in the study slightly favored using the template system. These results are supported by a prior study that measured satisfaction with the template in the ED 1 month and 1 year after implementation. This prior study found that satisfaction was weakly positive initially and further improved with continued use of the product. ${ }^{9}$ Further studies should address how continued use of the product affects satisfaction in the family medicine setting. In addition, physicians refer back to prior documented visits during patient follow-up. Physician satisfaction with a template system for this purpose should be studied.

The results of our study are consistent with a similar study within an emergency medicine setting. ${ }^{8}$ The Emergency Medicine Study also used the T-System and showed improved billing but a nonstatistically insignificant decrease in patient visit time. Physician satisfaction in the Emergency Medicine Study was higher than in our setting when measured after a longer period of use.

There are several limitations to this study and areas for further study. Both residents and faculty perceived the template documentation as slightly faster. Studying the exact time of physician evaluation rather than total patient visit time might detect a difference between the template system and the undirected format. Speed with the product might improve with continued use so repeating the 
measurements after longer exposure to the template might yield greater differences.

A further limitation of our study is that it did not index diagnoses or patient complaints. We assumed similar levels of complexity of care because new patients are assigned to either of the 2 teams and subsequently followed in the same team.

This study compared a template-driven medical documentation system to undirected written documentation. Further studies should compare template systems to other types of documentation methods including EMR. Both the residents and faculty are salaried without productivity incentives. There was no financial incentive for them to improve patient billing data. This may affect their satisfaction with the product. Studying a templatedriven documentation system in a family medicine setting where billing data affects physician reimbursement might yield improved satisfaction data.

This study looked at billing data but not reimbursement. Further study of charting methods should analyze the actual money generated related to improved billing data. A shift to billing at a higher level requires consideration of additional compliance issues. Medicare patients seen by family medicine residents must also be seen and examined by an attending physician for 99214 and 99215 levels of service. 9 Local practices may require changes to policy with additional surveillance that may affect the overall generated revenue.

This study focused on billing data, clinic visit time, and physician satisfaction. Further studies on medical documentation should include patient-oriented outcomes. These would include effects on patient satisfaction and on the patient-physician interaction.

The challenge of finding optimal documentation tools will continue in medicine. This study compared a template-driven medical documentation system to undirected written documentation in a family medicine residency clinic. The template system was preferred by the physicians, did not affect clinic times, and improved billing data. On study completion, we chose to implement the use of the template system throughout our clinic.

We thank the residents and staff at the Parkland Family Medicine Residency clinic for their support and participation in this study. SM was responsible for study design development, writing, and editing the manuscript. GS and CMC participated in study design development and editing; SR participated in revising and editing, along with providing professional expertise in the area of family medicine. BF was responsible for data analysis and editing, and AP participated in revising and editing and was responsible for study coordination.

\section{References}

1. Wrenn K, Rodewald L, Lumb E, Slovis C. The use of structured, complaint-specific patient encounter forms in the emergency department. Ann Emerg Med 1993;22:805-12.

2. Schriger DL, Baraff LJ, Rogers WH, Cretin S. Implementation of clinical guidelines using a computer charting system. JAMA 1997;278:1585-90.

3. Schmit J. Health care's paper trail is costly route. USA Today 2004 July 20;Sect. B:1.

4. Brailer D. The decade of health information technology: delivering consumer-centric and information-rich health care. Available from: http://www. hhs.gov/onchit/index.html.

5. Kikano GE, Goodwin MA, Sange KC. Evaluation and management services. A comparison of medical record documentation with actual billing in community family practice. Arch Fam Med 2000;9:68-71.

6. King MS, Sharp L, Lipsky MS. Accuracy of CPT evaluation and management coding by family physicians. J Am Board Fam Pract 2001;14:184-92.

7. King MS, Lipsky MS, Sharp L. Current procedural terminology coding: do the experts agree? J Am Board Fam Pract 2000;13:144-6.

8. Marill KA, Gaubarou ES, Nelson BK, Peterson MA, Gonzalez MR. Prospective, randomized trial of template-assisted versus undirected written recording of physician records in the emergency department. Ann Emerg Med 1999;33:500-9.

9. Centers for Medicare \& Medicaid Services, Carriers Manual, Fee Schedule for Physician's Services. Section 15016-Supervising physicians in teaching settings. November 2002. Available from: http://www. cms.hhs.gov/manuals/14_car/3b15000.asp\#_15016_0. 